\title{
Application of integrated management bundle incorporating with multidisciplinary measures improved in-hospital outcomes and early survival in geriatric hip fracture patients with perioperative heart failure: a retrospective cohort study
}

\author{
Mingming $\mathrm{Fu}^{1}$ · Yaqian Zhang ${ }^{1}$ · Junfei Guo ${ }^{2} \cdot$ Yuqi Zhao ${ }^{1}$. Zhiyong Hou ${ }^{2,3} \cdot$ Zhiqian Wang $^{1} \cdot$ Yingze Zhang $^{2,3,4}$
}

Received: 14 September 2021 / Accepted: 18 November 2021 / Published online: 24 January 2022

(c) The Author(s) 2021

\begin{abstract}
Background In elderly, hip fracture is often complicated by perioperative heart failure, related to worse prognosis. We aimed to analyze the effects of integrated management bundle incorporating with multidisciplinary measures on in-hospital outcomes and early survival in elderly hip fracture patients with perioperative heart failure.

Methods In this retrospective cohort study, a total of 421 hip fracture patients aged 65 and over who developed perioperative heart failure were included. According to different perioperative management modes applied, patients were retrospectively divided into multidisciplinary management group (Group A), including 277 patients, and integrated management bundle group (Group B), including 144 patients. The B-type natriuretic peptide (BNP) and C-reactive protein (CRP) levels, complications, length of stay, and hospitalization costs were observed and compared between two groups. Overall survival was compared by Kaplan-Meier methods. Cox regression analysis was used to identify prognostic factors associated with overall survival.

Results A total of 421 patients were enrolled for analysis, including 277 in Group A and 144 in Group B. BNP and CRP levels were significantly decreased compared with admission $(P<0.05)$. Furthermore, BNP and CRP in Group B decreased much more than those in Group A $(P<0.05)$. The reductions were observed in length of stay, hospitalization costs and incidence of pulmonary infection, hypoproteinemia, and acute cerebral infarction in Group B (all $P<0.05$ ). The Kaplan-Meier plots showed significantly superior overall survival in Group B. Integrated management bundle was independent favorable prognostic factors.

Conclusions The integrated management bundle incorporating with multidisciplinary measures significantly improved the therapeutic effect of perioperative heart failure, reduced inflammatory response, and yielded better hospital outcomes. It brought better survival benefits for geriatric hip fracture patients with perioperative heart failure. The results of this study can play an important role in clinical work and provide a valuable theoretical basis for selection of management model in elderly hip fracture patients with perioperative heart failure.
\end{abstract}

Keywords Hip fracture $\cdot$ Geriatric $\cdot$ Perioperative heart failure $\cdot$ Complications $\cdot$ Survival $\cdot$ Management bundle

Mingming Fu and Yaqian Zhang contributed equally to this work.

Zhiqian Wang

w18533112890@163.com

Yingze Zhang

dryzzhang@126.com

Extended author information available on the last page of the article

\section{Introduction}

Due to rapidly aging populations, the number of elderly hip fracture patients is increasing [1,2]. Hip fractures commonly result in loss of functionality, disability, and increase mortality in elderly, which has become a global medical and health problem [3]. In such case, effective interventions are needed to improve the function and prognosis of elderly hip fracture patients. A recent study by Invernizzi et al. [4] showed that a multidisciplinary rehabilitative could improve function and 
reduce disability in hip fracture patients. Gamboa-Arango et al. [5] reported that better functionality at the hospital discharge improved the prognosis of elderly hip fracture patients. Trevisan et al. [6] believed enhanced patient management, aggressive rehabilitation might further reduce mortality of elderly hip fracture patients.

Heart failure is one of the most frequent perioperative complications in elderly patients with hip fractures and an important risk factor for mortality after hip surgery [7-12]. The mechanism of perioperative heart failure is extremely complex. Geriatric hip fracture patients are in a state of persistent stress, because of fear, pain, anxiety, and blood loss, leading to increased myocardial oxygen consumption and imbalance between myocardial oxygen supply and demand, followed by perioperative myocardial ischemia and injury. This condition, when severe, usually leads to myocardial stunning and heart failure [13-15]. Due to the prevalence and complexity of heart failure, perioperative heart failure management has become a major challenge for physicians $[16,17]$. Although much attention has been paid to the perioperative management in elderly hip fracture patients, further research is needed to explore a better management model in elderly hip fracture patients with perioperative heart failure. On the basis of clinical practice and experiences, we find that integrated management bundle incorporating with multidisciplinary measures is more suitable for elderly hip fracture patients with perioperative heart failure.

We aimed to analyze the effects of integrated management bundle incorporating with multidisciplinary measures on in-hospital outcomes and early survival in elderly hip fracture patients with perioperative heart failure. We hypothesized that this new type of management model was associated with lower B-type natriuretic peptide (BNP) level, lower complication rates, fewer total hospitalization costs, shorter hospital length of stay, and better survival.

\section{Material and methods}

\section{Patients and groups}

The retrospective cohort study was based on data collected from Jan, 2017, to Sept, 2019, at a single Level I trauma center in China. The study protocol was approved by the institutional review board of the third Hospital of Hebei Medical University, and an exemption from the informed consent was obtained. Inclusion criteria were hip fracture patients aged 65 years and over with perioperative heart failure, who had a delay less than 1 week from injury to admission, and underwent hip surgery. Diagnosis of heart failure should be based on clinical signs, symptoms, and prior cardiovascular history and further confirmed by appropriate additional investigations, such as BNP, electrocardiogram, chest X-ray, and echocardiography [18]. Besides, all patients enrolled were treated with common drugs for heart failure, and blood samples were collected at least right after admission as well as just before discharge to determining BNP and C-reactive protein (CRP). Exclusion criteria were multiple fractures or injuries, pathological fractures, chronic heart failure, malignancy, autoimmune disease, and preexisting systemic inflammatory comorbidity. The patients who admitted to our department from Jan 2017 to Sept 2019, and met the inclusion criteria, with no exclusion criteria, were included in this analysis. According to different perioperative management modes applied, patients were retrospectively divided into multidisciplinary management group (Group A) and integrated management bundle group (Group B).

\section{Perioperative management}

Different from most hospitals, our hospital has a specialist ward for geriatric orthopedics staffed by orthopedists, internists, rehabilitation physicians, and trained nursing personnel, that provides centralized management and sustainable $24 / 7 / 365$ geriatric support. The multidisciplinary team for geriatric fracture participates in the ward round 7 days a week. The patients are assessed by at least two orthopedists who lead the team and make the preparation related to the surgical procedure, along with an internist who is available to review perioperative care for patients with comorbidities, in cooperation with an anesthetic consultant. Rehabilitation physicians guide the patients in rehabilitation care. In the first stage, multidisciplinary management was applied to elderly hip fracture patients with perioperative heart failure.

Depending on clinical experience and related guidelines, the team optimized the original protocol, proceeding to a new stage of perioperative management, which is the second stage. In the second stage, considering the characteristics of the elderly hip fracture patients and the unique medical system in China, we simplified, optimized, and integrated the existing management model to form the integrated management bundle incorporating with multidisciplinary measures, so that it is more in line with the clinical actual situation and more feasible. The core members of the team include orthopedic specialists, internal medicine specialist with well-recognized skills in geriatric medicine, anesthesiologists, rehabilitation physicians, and specialist nurses. From the time of hospital admission onwards, hip fracture patients were given the integrated management bundle, including monitoring, evaluation and education, respiratory management, volume management, nutritional support, blood and thrombosis management, sedation and analgesia, and tube management. The internist with well-recognized skills in geriatric medicine focused on the comprehensive assessment of diseases in multiple systems on the basis of a holistic 
view, without relying on clinical consultations. The specific measures were described in more detail below.

Group A was in the first stage. In the course of multidisciplinary management, the monitoring of electrocardiogram, mean arterial pressure, and pulse oxygen saturation was performed in patients with severe comorbidities, which guaranteed the detection and management of cardiovascular and cerebrovascular complications promptly $[19,20]$. Oxygen treatment was restricted to patients with pulmonary infection or respiratory failure. Assessment of nutritional status was not routinely recommended, only if the patient recently lost weight or had a low body mass index on admission. Nutrition therapy was only available for a subset of patients. Food was not allowed to take within $8 \mathrm{~h}$ before surgery. The analgesics, including either an opioid, a nonsteroidal anti-inflammatory drug (NSAID), or acetaminophen, were applied to ease pain. Low-molecular-weight heparin and ankle pump exercise were administered to prevent deep vein thrombosis. Patients suspected of urinary retention were given single catheterization. Indwelling time of urethral catheter took for several days, in case of persistent urinary retention [21].

Group B was in the second stage. Patients in Group B were applied to integrated management bundle incorporating with multidisciplinary measures, including monitoring, evaluation and education, respiratory management, volume management, nutritional support, blood and thrombosis management, sedation and analgesia, and tube management. The monitoring of electrocardiogram, mean arterial pressure, and pulse oxygen saturation was suitable for all patients. Once admitted, patients were assessed by comprehensive geriatric assessment [22], which could detect potential risks and prompt intervention immediately. The health care should be effectively preached and there was an opportunity to establish harmonious relationship with patients and their families. Respiratory management was listed as follows: chest physiotherapy and breathing exercises were required, including actively cough, accessary posture productive cough, and turnover [23]. Low-flow inhale oxygen and atomization were indispensable measures. The patient was treated with nebulization of salbutamol sulfate, ipratropium bromide, and budesonide twice daily. Patients had a documented order for strict monitoring of fluid intake and output, to achieve the negative fluid balance [24]. All of the patients were evaluated for nutritional status on admission, and nutrition therapy was started in accordance with specific conditions [25]. Probiotics and prokinetic agents were used to avoid acute gastrointestinal dysfunction. Oral feeding was the preferred method, and if nutrient intake was insufficient, early detaining gastric tube was chosen to avoid electrolyte imbalance. We recommended that patients maintained a hemoglobin level of at least $10 \mathrm{~g}$ per deciliter of blood. The basic, physical, and pharmacological prevention measures were actively applied to prevent the deep vein thrombosis of lower extremities [26]. For perioperative agitation and delirium, attempts should be made to identify and relieve the likely causative triggers, beyond just those drug treatments. Pain management focused on the use of multimodal analgesia, which included effective early analgesia, the application of acetaminophen and nonsteroidal anti-inflammatory medication, and patient-controlled analgesia [27]. Patients suspected of urinary retention were given ultrasound bladder scan. Urinary retention was treated with a single catheterization at first, and the second remained urethral catheter in place for 1-2 days[21].

\section{Data collection}

Clinical data and baseline variables of all patients were collected from the patients' electronic medical records. The following information was extracted: gender, age, mechanisms of injury, comorbidities, fracture type (femoral neck fracture or intertrochanteric fracture), surgery type (fixation or replacement), anesthesia type (general or regional), intraoperative blood loss, duration of operation, complications, BNP (in units of $\mathrm{pg} / \mathrm{mL}$ ), CRP (in units of $\mathrm{mg} / \mathrm{L}$ ), admission delay time, hospital length of stay, and total hospitalization costs. The follow-up started at the date of enrollment in the cohort and the endpoint was the date of death or the end of the study, whichever came first. The primary outcomes included BNP measured before discharge and overall survival at 2 years. The secondary outcomes included the rates of other perioperative complications, hospital length of stay, and total hospitalization costs.

C-reactive protein is one of the most representative markers in the acute phase of systemic inflammatory response, which has been found to be associated with coronary heart disease and heart failure [28, 29]. Whether there was a correlation between CRP and BNP in elderly hip fracture patients with perioperative heart failure has not been studied. Moreover, it was convenient to dynamically monitor CRP levels clinically. Thus, we used CRP as inflammatory marker.

\section{Statistical analysis}

The continuous variables were evaluated for normality by using the Shapiro-Wilk test. Normally distributed variables were presented as mean and standard deviation (SD); otherwise, they are presented as median and interquartile range. Categorical variables were presented as numbers and percentages. Student's $t$-test or Mann-Whitney $U$ test was used to compare differences between groups for continuous variables as appropriate, while the Chi-square test or Fisher exact test for categorical variables. The correlation of BNP and CRP was evaluated by Spearman's correlation and statistical significance. Kaplan-Meier method was used to estimate the survival, and any difference in survival was 
evaluated with a log-rank test. Univariate and multivariate analyses of survival outcomes were conducted with the Cox proportional hazards regression model, to determine the independent prognostic factors. All the statistical analyses and graphics were performed with the SPSS statistical software (version 26.0) and R statistical software (version 3.6.3). The level of significance was set at $P<0.05$.

\section{Results}

\section{Patient characteristics}

From Jan 2017 to Sept 2019, a total of 476 geriatric patients with hip fracture were screened to participate in this study. Among them, 55 patients were eliminated by the exclusion criteria, and the remaining 421 were finally analyzed. Specifically, 10 patients had multiple fractures or injuries, or pathological fractures; 18 patients received nonsurgical treatment; 27 patients admitted with a delay of more than 7 days (Fig. 1). A total of 421 patients met inclusion and were included in our analysis, of which 277 received multidisciplinary management and 144 underwent integrated management bundle. A majority of patients was female $(70.8 \%)$ and mean age was 81.8 years (SD 7.1). The average follow-up was 21 months. The clinical characteristics of patients in two groups are presented in Table 1, which did not differ significantly.

\section{Comparison of complications and hospital outcomes}

The comparison of perioperative complications and hospital outcomes is shown in Table 2. In terms of type of complications, Group B had a reduction in incidence of pulmonary infection, acute cerebral infarction, and hypoproteinemia ( $P=0.023, P=0.022$, and $P=0.009$, respectively). No differences were found in the incidence of arrhythmia, electrolyte disturbance, deep venous thrombosis of the lower limbs, and urinary infection between two groups. BNP and CRP values for both the two groups decreased postmanagement $(P<0.001)$. There was a greater decrease of BNP and CRP values in Group B as compared to Group A ( $P=0.011$, and $P=0.010$, respectively). Moreover, integrated management bundle could shorten hospital length of stay and reduce total hospitalization costs than routine management (Table 2).

\section{The correlations between BNP and CRP levels}

Scatterplot presenting the correlations between BNP and CRP levels is shown in Fig. 2. There was a moderate positive correlation between admission BNP and admission CRP levels $(r=0.500, P<0.001)$. Likewise, Spearman correlation between discharge BNP and discharge CRP showed a moderate positive correlation $(r=0.600$, $P<0.001)$.

Fig. 1 The flow diagram of this study

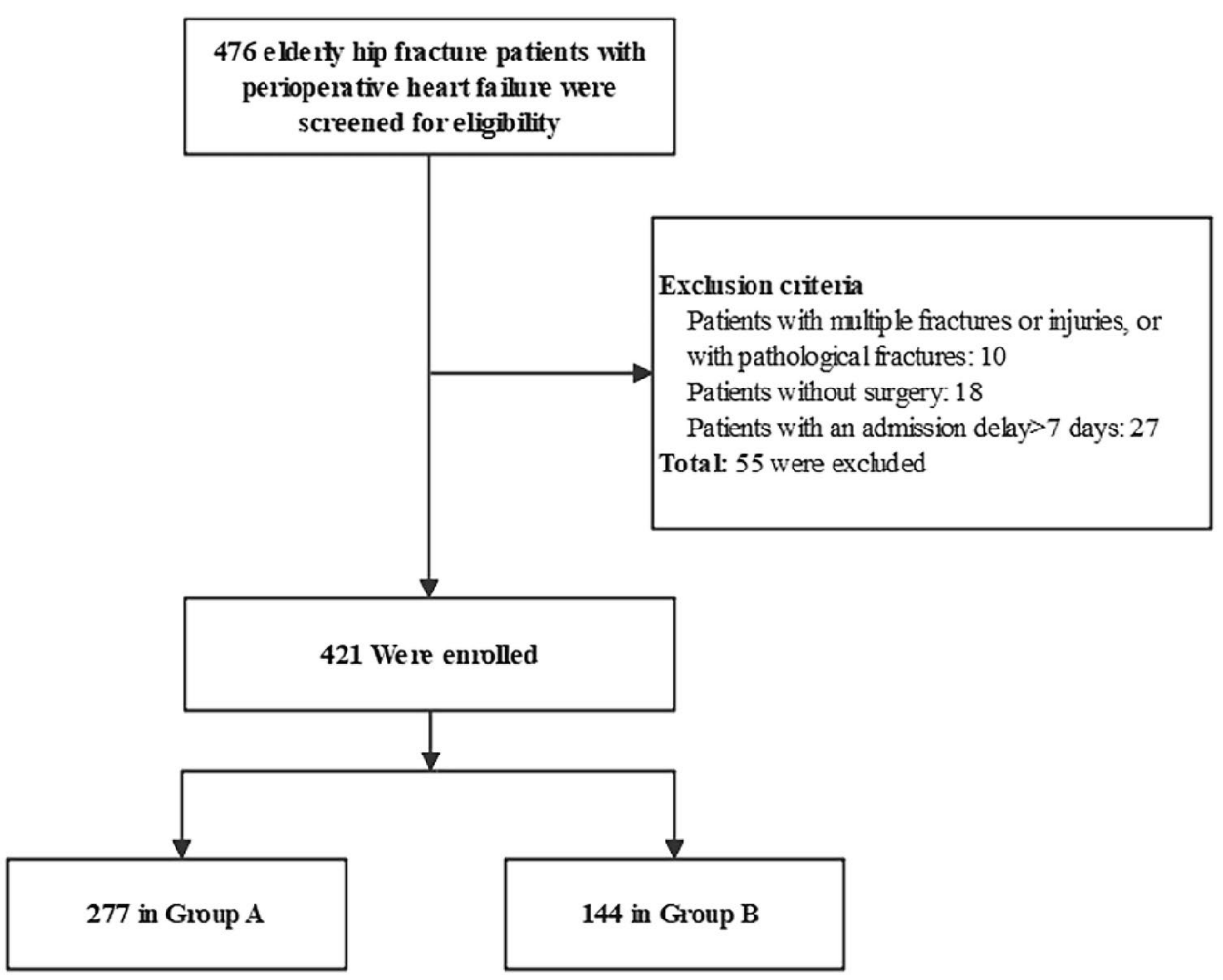


Table 1 Baseline characteristics of geriatric hip fracture patients with perioperative heart failure

\begin{tabular}{|c|c|c|c|c|c|}
\hline & $\begin{array}{l}\text { Total } \\
(n=421)\end{array}$ & $\begin{array}{l}\text { Group A } \\
(n=277)\end{array}$ & $\begin{array}{l}\text { Group B } \\
(n=144)\end{array}$ & $\chi^{2} / t / Z$ & $P$ value \\
\hline \multicolumn{6}{|l|}{ Gender, $n(\%)$} \\
\hline Male & $123(29.2 \%)$ & $80(28.9 \%)$ & $43(29.9 \%)$ & \multirow[t]{2}{*}{0.044} & \multirow[t]{2}{*}{0.834} \\
\hline Female & $298(70.8 \%)$ & $197(71.1 \%)$ & $101(70.1 \%)$ & & \\
\hline Age, mean \pm SD (years) & $81.8 \pm 7.1$ & $81.8 \pm 7.4$ & $81.7 \pm 6.5$ & 0.192 & 0.848 \\
\hline \multicolumn{6}{|l|}{ Age group, $n(\%)$} \\
\hline$<80$ years & $151(35.9 \%)$ & $100(36.1 \%)$ & $51(35.4 \%)$ & \multirow[t]{2}{*}{0.019} & \multirow[t]{2}{*}{0.890} \\
\hline$\geq 80$ years & $270(64.1 \%)$ & $177(63.9 \%)$ & $93(64.6 \%)$ & & \\
\hline BMI (normal/overweight/obesity) & $272 / 115 / 34$ & $177 / 76 / 24$ & $95 / 39 / 10$ & 0.414 & 0.813 \\
\hline \multicolumn{6}{|l|}{ Mechanism of injury, $n(\%)$} \\
\hline Low energy & $408(96.9 \%)$ & $265(95.7 \%)$ & $143(99.3 \%)$ & \multirow[t]{2}{*}{3.062} & \multirow[t]{2}{*}{0.080} \\
\hline High energy & $13(3.1 \%)$ & $12(4.3 \%)$ & $1(0.7 \%)$ & & \\
\hline \multicolumn{6}{|l|}{ Fracture types, $n(\%)$} \\
\hline Femoral neck fractures & $193(45.8 \%)$ & $131(47.3 \%)$ & $62(43.1 \%)$ & \multirow[t]{2}{*}{0.685} & \multirow[t]{2}{*}{0.408} \\
\hline Intertrochanteric fractures & $228(54.2 \%)$ & $146(52.7 \%)$ & $82(56.9 \%)$ & & \\
\hline \multicolumn{6}{|l|}{ Comorbidities, $n(\%)$} \\
\hline Hypertension & $214(50.8 \%)$ & $146(52.7 \%)$ & $68(47.2 \%)$ & 1.141 & 0.286 \\
\hline Coronary heart disease & $121(28.7 \%)$ & $83(30.0 \%)$ & $38(26.4 \%)$ & 0.591 & 0.442 \\
\hline Diabetes & $92(21.9 \%)$ & $62(22.4 \%)$ & $30(20.8 \%)$ & 0.133 & 0.715 \\
\hline Cerebrovascular disease & $157(37.3 \%)$ & $106(38.3 \%)$ & $51(35.4 \%)$ & 0.329 & 0.566 \\
\hline \multicolumn{6}{|l|}{ Surgical type, $n(\%)$} \\
\hline Replacement & $183(43.5 \%)$ & $125(45.1 \%)$ & $58(40.3 \%)$ & \multirow[t]{2}{*}{0.906} & \multirow[t]{2}{*}{0.341} \\
\hline Fixation & $238(56.5 \%)$ & $152(54.9 \%)$ & $86(59.7 \%)$ & & \\
\hline \multicolumn{6}{|l|}{ Anesthesia type, $n(\%)$} \\
\hline General & $201(47.7 \%)$ & $133(48.0 \%)$ & $68(47.2 \%)$ & \multirow[t]{2}{*}{0.024} & \multirow[t]{2}{*}{0.877} \\
\hline Regional & $220(52.3 \%)$ & $144(52.0 \%)$ & $76(52.8 \%)$ & & \\
\hline Intraoperative blood loss & $200(200,300)$ & $200(200,300)$ & $200(200,300)$ & -0.568 & 0.570 \\
\hline Duration of operation & $108.6 \pm 30.8$ & $110.0 \pm 30.2$ & $105.9 \pm 31.8$ & 1.294 & 0.196 \\
\hline Admission BNP & $304(233,444)$ & $290(225,457)$ & $327(249,427)$ & 1.515 & 0.130 \\
\hline Admission CRP & $56.1(37.4,84.2)$ & $56.3(36.8,81.6)$ & $55.7(38.5,88.3)$ & 0.360 & 0.719 \\
\hline
\end{tabular}

Values are presented as mean \pm standard deviation, median (P25, P75), or number (percentage) as appropriate. $S D$ standard deviation, $B M I$ body mass index, $B N P$ B-type natriuretic peptide, $C R P \mathrm{C}$-reactive protein. $\chi^{2}$ value is Chi-square test statistic, and $t$ value is $t$-test statistic. $Z$ is the $Z$-score for Wilcoxon test

\section{Survival analysis for patients}

Compared with multidisciplinary management group, the 2-year mortality was significantly lower in integrated management bundle group (20.9\% vs $12.5 \%, P=0.033)$. Kaplan-Meier survival curves for two groups are shown in Fig. 3. A comparison of survival curves using log-rank test indicated that integrated management bundle significantly improved survival $(P=0.034)$.

\section{Identify prognostic factors}

We employed Cox proportional hazards model to analyze possible prognostic factors associated with overall survival. In univariate Cox model, the significant variables were age $\geq 80$, comorbidities $\geq 2$, and received integrated management bundle $(P<0.05)$. Multivariate analysis revealed age $\geq 80$ and comorbidities $\geq 2$ were independent risk factors for poor prognosis (HR $>1, P<0.05)$. On the contrary, integrated management bundle was independent favorable prognostic factors $(\mathrm{HR}<1, P<0.05)$ (Table 3 ). 
Table 2 Comparisons of perioperative complications and hospital outcomes between two groups

Table 3 Cox proportional hazards regression model for overall survival

\begin{tabular}{lllrl}
\hline Variables & $\begin{array}{l}\text { Group A } \\
(n=277)\end{array}$ & $\begin{array}{l}\text { Group B } \\
(n=144)\end{array}$ & $\chi^{2} / t / Z$ & $P$ value \\
\hline $\begin{array}{l}\text { Perioperative complications } \\
\text { Pulmonary infection }\end{array}$ & $120(43.3 \%)$ & $46(31.9 \%)$ & 5.135 & $0.023^{*}$ \\
Arrhythmia & $96(34.7 \%)$ & $49(34.0 \%)$ & 0.017 & 0.897 \\
Acute cerebral infarction & $14(5.1 \%)$ & $1(0.7 \%)$ & 5.241 & $0.022^{*}$ \\
Hypoproteinemia & $212(76.5 \%)$ & $93(64.6 \%)$ & 6.779 & $0.009 *$ \\
Electrolyte disturbance & $209(75.5 \%)$ & $104(72.2 \%)$ & 0.518 & 0.472 \\
Deep venous thrombosis of & $134(48.4 \%)$ & $63(43.8 \%)$ & 0.814 & 0.367 \\
$\quad$ the lower limbs & $6(2.2 \%)$ & $7(4.9 \%)$ & & 1.487 \\
$\quad$ Urinary infection & $162(98,273)$ & $128(94,191)$ & -2.547 & 0.233 \\
Discharge BNP & $31.5(16.0,49.6)$ & $25.8(14.1,39.1)$ & -2.589 & $0.011^{*}$ \\
Discharge CRP & $15.1 \pm 5.3$ & $13.2 \pm 4.9$ & 3.416 & $0.010^{*}$ \\
Length of stay & $7.2 \pm 2.0$ & $6.7 \pm 2.0$ & 2.606 & $0.010^{*}$ \\
Total hospitalization costs & & & & \\
\hline
\end{tabular}

Values are presented as mean \pm standard deviation, median (P25, P75), or number (percentage) as appropriate. *Statistically significant. BNP B-type natriuretic peptide, CRP C-reactive protein. $\chi^{2}$ value is Chisquare test statistic, and $t$ value is $t$-test statistic. $Z$ is the $Z$-score for Wilcoxon test

\begin{tabular}{|c|c|c|c|c|}
\hline \multirow[t]{2}{*}{ Variables } & \multicolumn{2}{|l|}{ Univariate Cox } & \multicolumn{2}{|l|}{ Multivariate Cox } \\
\hline & $\operatorname{HR}(95 \% \mathrm{CI})$ & $P$ value & $\mathrm{HR}(95 \% \mathrm{CI})$ & $P$ value \\
\hline \multicolumn{5}{|l|}{ Gender } \\
\hline Female & Reference & 0.894 & Reference & 0.649 \\
\hline Male & $1.034(0.634-1.687)$ & & $1.121(0.684-1.838)$ & \\
\hline \multicolumn{5}{|l|}{ Age group } \\
\hline$<80$ years & Reference & $0.007 *$ & Reference & $0.003 *$ \\
\hline$\geq 80$ years & $2.090(1.218-3.584)$ & & $2.331(1.337-4.063)$ & \\
\hline \multicolumn{5}{|l|}{ Mechanism of injury } \\
\hline Low energy & Reference & 0.779 & Reference & 0.924 \\
\hline High energy & $0.818(0.201-3.331)$ & & $0.933(0.225-3.871)$ & \\
\hline \multicolumn{5}{|l|}{ Comorbidities } \\
\hline $0-2$ & Reference & $0.018^{*}$ & Reference & $0.008 *$ \\
\hline$>2$ & $1.808(1.108-2.949)$ & & $1.963(1.196-3.224)$ & \\
\hline \multicolumn{5}{|l|}{ Fracture type } \\
\hline Femoral neck & Reference & 0.692 & Reference & 0.065 \\
\hline Intertrochanteric & $0.913(0.582-1.432)$ & & $0.392(0.145-1.058)$ & \\
\hline \multicolumn{5}{|l|}{ Surgery type } \\
\hline Replacement & Reference & 0.865 & Reference & 0.125 \\
\hline Fixation & $1.040(0.660-1.640)$ & & $2.189(0.804-6.010)$ & \\
\hline \multicolumn{5}{|l|}{ Anesthesia type } \\
\hline Regional & Reference & 0.133 & Reference & 0.231 \\
\hline General & $0.689(0.435-1.092)$ & & $0.753(0.473-1.199)$ & \\
\hline \multicolumn{5}{|l|}{ Management mode } \\
\hline Multidisciplinary management & Reference & $0.037 *$ & Reference & $0.047^{*}$ \\
\hline Integrated management bundle & $0.570(0.336-0.968)$ & & $0.581(0.341-0.992)$ & \\
\hline
\end{tabular}

*Statistically significant 
Fig. 2 Scatterplot of the correlations between B-type natriuretic peptide and $\mathrm{C}$-reactive protein levels
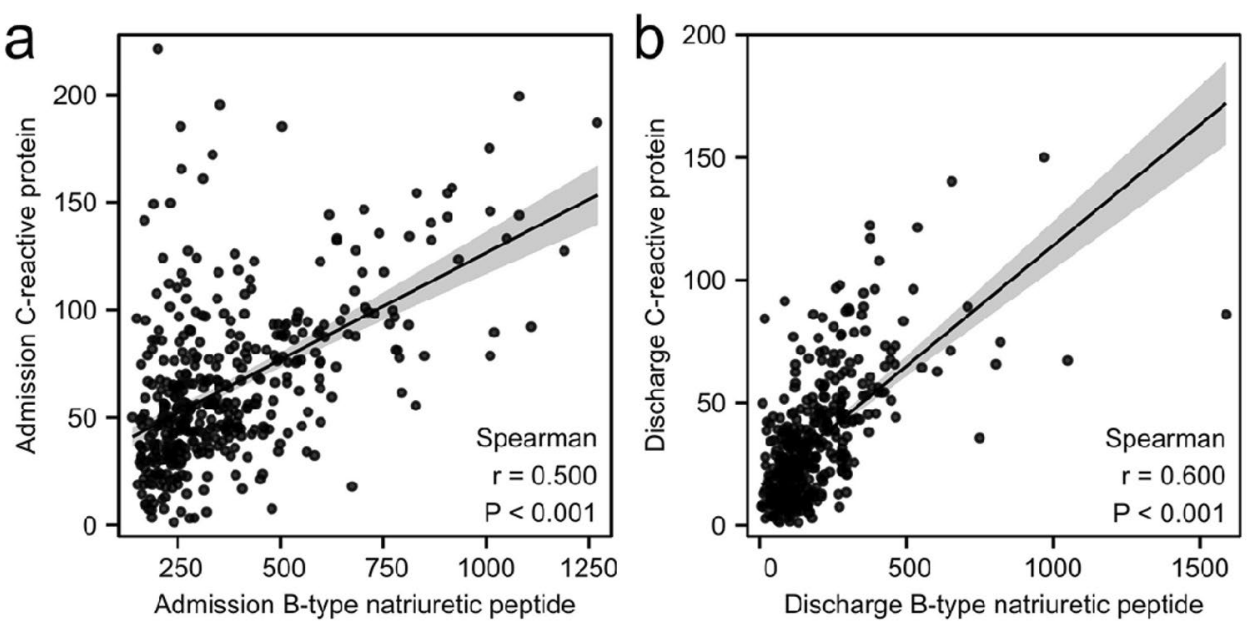

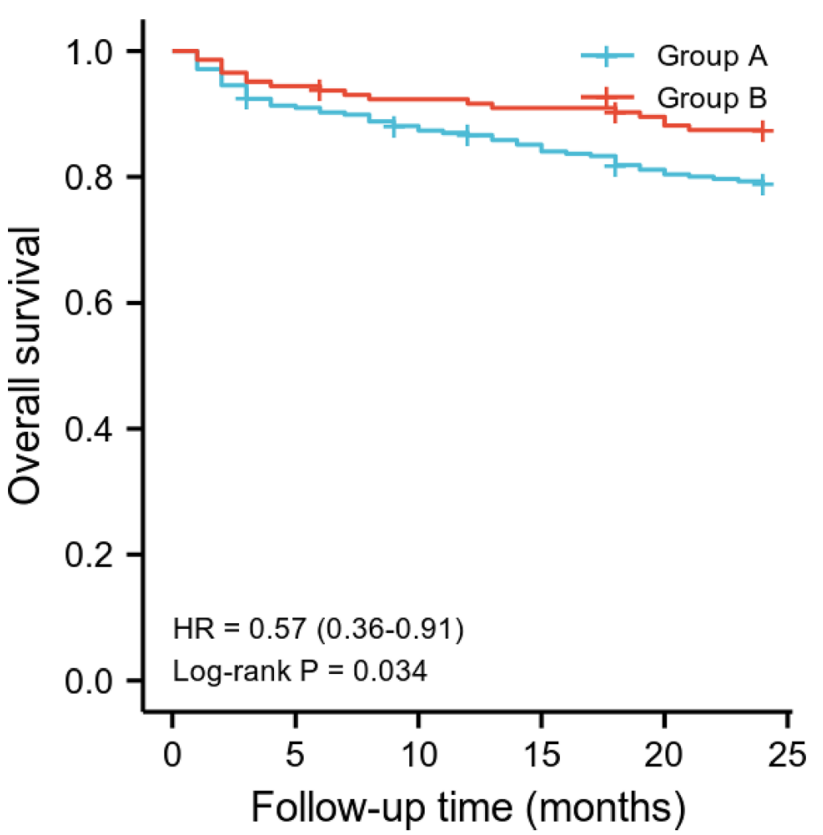

Fig. 3 Kaplan-Meier survival curves for elderly patients with perioperative heart failure

\section{Discussion}

In this retrospective study, we analyzed the clinical characteristics and prognosis of geriatric hip fracture patients with perioperative heart failure. In our results, the application of integrated management bundle incorporating with multidisciplinary measures had been found to be associated with lower BNP and CRP levels, lower complication rates, including pulmonary infection, acute cerebral infarction, and hypoproteinemia, fewer total hospitalization costs, shorter length of stay, and better survival. There was a moderate positive correlation between BNP and CRP levels.
Hip fracture patients are often of advanced age and suffer from multiple comorbidities [30], which poses challenges for clinical diagnosis and treatment. As a result, orthogeriatric management model comes into existence, which is the multidisciplinary collaboration model for older patients with orthopedic disorders. So far there are some consensus and established protocols about orthogeriatric management [27, 31-33]. In combination with the characteristics of the elderly hip fracture patients and the unique medical system in China, the management model is simplified, optimized, and integrated to form the integrated management bundle incorporating with multidisciplinary measures, so that it is more in line with the clinical actual situation and more feasible. Heart failure is one of the common and serious perioperative complications in elderly hip fracture patients [34]. Nevertheless, high-quality studies are still scarce, which is about the management on geriatric hip fracture patients with perioperative heart failure.

It is difficult to make an early diagnosis of heart failure based on symptoms and signs alone, which are often unspecific and similar to those of other diseases. In such clinical situations, BNP is often superior to clinical diagnosis for diagnosing heart failure [16]. Before discharge, BNP and CRP levels were significantly decreased compared with admission. Furthermore, BNP and CRP levels in integrated management bundle group decreased much more than that of multidisciplinary management group. The mechanism of perioperative heart failure is extremely complex. Specifically, stress triggers the activation of sympathetic nervous system, which mediates the release of catecholamines and glucocorticoids, increasing sodium retention, vasoconstriction, and cardiac workload, and ultimately leading to perioperative myocardial ischemia and injury. Another possible mechanism is that an abundant increase in inflammatory factors caused by stress leads to perioperative myocardial ischemia and injury. As diagnostic and prognostic markers of heart failure 
[35], brain natriuretic peptide (BNP) is secreted from the heart in response to stretch and stress. Raised levels of BNP positively correlate with the severity of heart failure [36-38]. C-reactive protein is a nonspecific indicator of the inflammatory response, applied to assess the severity of traumatic stress.

Medication remains the cornerstone of therapy for heart failure. Nevertheless, application of high-quality and effective management interventions plays a crucial role in promoting recovery and improving outcomes based on pharmacotherapy. According to the results of this study, the reduction was also observed in incidence of pulmonary infection, hypoproteinemia, and acute cerebral infarction in integrated management bundle group. We highlight the bundled application of numerous measures, especially respiratory management, volume management, nutritional support, and blood management in integrated management bundle group, which reduced traumatic stress and inflammatory responses much more than those in multidisciplinary group.

Recently, Zhang et al. discovered that the levels of plasma BNP were positively correlated with CRP levels in patients with sepsis [39]. A similar phenomenon was also observed in the present study. Various studies have shown that inflammatory response plays an important role in pathogenesis of heart failure, associated with the severity of traumatic stress. $\mathrm{C}$-reactive protein is a nonspecific indicator of the inflammatory response, influenced by many factors, including surgical trauma, fracture, infection, and tumor [40-42]. In this study, we excluded patients with systemic inflammatory diseases and malignant tumors, to minimize other factors of influence on our results. Our study was a direct demonstration of correlation between perioperative heart failure and inflammation response in geriatric hip fracture patients. Nevertheless, the prognostic and diagnostic value of CRP required further investigation in geriatric hip fracture patients with perioperative heart failure.

The results showed that the integrated management bundle exerted significant survival benefits for geriatric hip fracture patients with perioperative heart failure in overall survival curves. Multivariate Cox regression analysis determined integrated management bundle was independent favorable prognostic factors. Neuerburg et al. [43] demonstrated that interdisciplinary orthogeriatric management improved the long-term outcome of hip fracture patients. Liu and Rosas et al. [25] presented clinical evidence demonstrating that enhanced recovery after surgery (ERAS) was associated with significant decreases in hospital length of stay and postoperative complication and it is effective in improving outcomes in surgical populations. That is to say, optimization of original perioperative management mode could improve outcomes of patients [20, 44-46]. Further studies should be carried out in order to better clarify the effect. These bundled measures should be considered as first steps in the development of a more polished set of measures, and studies on perioperative management have never ceased.

\section{Limitations and strengths}

There are several limitations in this study. It is a singlecenter, retrospective cohort study and the intrinsic limitation of design seems to be inescapable. There is likely to be a selection bias due to lack of randomization. The variables involved in this study are limited to previously collected data. Moreover, the limited statistical power caused by small samples and short follow-up periods implies that the results should be interpreted with caution. The absence of standardization of the drugs administered among the elderly hip fracture patients with perioperative heart failure could be also viewed as a limitation of this study. Despite these limitations, this study has areas of strength. We provide clues on perioperative management of elderly hip fracture patients with perioperative heart failure.

\section{Conclusions}

The integrated management bundle incorporating with multidisciplinary measures significantly improved the therapeutic effect of perioperative heart failure, reduced perioperative inflammatory response, and yielded better hospital outcomes. It brought better survival benefits for geriatric hip fracture patients with perioperative heart failure. The results of this study can play an important role in clinical work and provide a valuable theoretical basis for selection of management model in elderly hip fracture patients with perioperative heart failure.

\section{Acknowledgements None.}

Funding This study was supported by The Natural Science Foundation of Hebei Province (Grant no. H2019206046).

Data availability The data used to support the findings of this study are available from Zhiqian Wang upon request.

\section{Declarations}

Conflict of interest The authors declare that there is no conflict of interest regarding the publication of this paper.

Statement of human and animal rights This study was carried out in respect of human and animal rights.

Ethical approval and Informed consent This study was approved by the institutional review board of the third Hospital of Hebei Medical 
University in compliance with the Helsinki and an exemption from the informed consent was obtained. All data were anonymized before the analysis to safeguard patient privacy.

Open Access This article is licensed under a Creative Commons Attribution 4.0 International License, which permits use, sharing, adaptation, distribution and reproduction in any medium or format, as long as you give appropriate credit to the original author(s) and the source, provide a link to the Creative Commons licence, and indicate if changes were made. The images or other third party material in this article are included in the article's Creative Commons licence, unless indicated otherwise in a credit line to the material. If material is not included in the article's Creative Commons licence and your intended use is not permitted by statutory regulation or exceeds the permitted use, you will need to obtain permission directly from the copyright holder. To view a copy of this licence, visit http://creativecommons.org/licenses/by/4.0/.

\section{References}

1. Edelmuth S, Sorio GN, Sprovieri FAA et al (2018) Comorbidities, clinical intercurrences, and factors associated with mortality in elderly patients admitted for a hip fracture. Rev Bras Ortop 53:543-551. https://doi.org/10.1016/j.rboe.2018.07.014

2. Guzon-Illescas O, Perez Fernandez E, Crespi Villarias $\mathrm{N}$ et al (2019) Mortality after osteoporotic hip fracture: incidence, trends, and associated factors. J Orthop Surg Res 14:203. https://doi.org/ 10.1186/s13018-019-1226-6

3. Johnell O, Kanis J (2005) Epidemiology of osteoporotic fractures. Osteoporos Int 16:S3-7. https://doi.org/10.1007/ s00198-004-1702-6

4. Invernizzi M, de Sire A, D'Andrea F et al (2019) Effects of essential amino acid supplementation and rehabilitation on functioning in hip fracture patients: a pilot randomized controlled trial. Aging Clin Exp Res 31:1517-1524. https://doi.org/10.1007/ s40520-018-1090-y

5. Gamboa-Arango A, Duaso E, Malafarina V et al (2020) Prognostic factors for discharge to home and residing at home 12 months after hip fracture: an Anoia hip study. Aging Clin Exp Res 32:925-933. https://doi.org/10.1007/s40520-019-01273-9

6. Trevisan C, Gallinari G, Klumpp R et al (2019) Year to year comparison of 2000-2015 in hip fracture management: same survival rate despite older and more fragile patients. Aging Clin Exp Res 31:1097-1103. https://doi.org/10.1007/s40520-018-1047-1

7. Cha YH, Ha YC, Ryu HJ et al (2020) Effect of heart failure on postoperative short and long-term mortality in elderly patients with hip fracture. Injury 51:694-698. https://doi.org/10.1016/j. injury.2020.01.004

8. Acan AE, Ozlek B, Kilinc CY et al (2020) Perioperative outcomes following a hip fracture surgery in elderly patients with heart failure with preserved ejection fraction and heart failure with a mid-range ejection fraction. Ulus Travma Acil Cerrahi Derg 26:600-606. https://doi.org/10.14744/tjtes.2020.23946

9. Bohsali F, Klimpl D, Baumgartner R et al (2020) Effect of heart failure with preserved ejection fraction on perioperative outcomes in patients undergoing hip fracture surgery. J Am Acad Orthop Surg 28:e131-e138. https://doi.org/10.5435/JAAOS-D-18-00731

10. Gerber Y, Melton LJ 3rd, Weston SA et al (2011) Osteoporotic fractures and heart failure in the community. Am J Med 124:418425. https://doi.org/10.1016/j.amjmed.2010.11.029

11. Cullen MW, Gullerud RE, Larson DR et al (2011) Impact of heart failure on hip fracture outcomes: a population-based study. J Hosp Med 6:507-512. https://doi.org/10.1002/jhm.918
12. Marco-Martinez J, Bernal-Sobrino JL, Fernandez-Perez $\mathrm{C}$ et al (2021) Impact of heart failure on in-hospital outcomes after surgical femoral neck fracture treatment. J Clin Med. https://doi.org/ 10.3390/jcm 10050969

13. Weber B, Lackner I, Gebhard F et al (2021) Trauma, a matter of the heart-molecular mechanism of post-traumatic cardiac dysfunction. Int J Mol Sci. https://doi.org/10.3390/ijms22020737

14. Wall J, Naganathar S, Praditsuktavorn B et al (2019) Modeling cardiac dysfunction following traumatic hemorrhage injury: impact on myocardial integrity. Front Immunol 10:2774. https:// doi.org/10.3389/fimmu.2019.02774

15. Lackner I, Weber B, Haffner-Luntzer M et al (2021) Systemic and local cardiac inflammation after experimental long bone fracture, traumatic brain injury and combined trauma in mice. J Orthop Translat 28:39-46. https://doi.org/10.1016/j.jot.2020.12.003

16. Tamamura Y, Matsuura M, Shiba S et al (2020) Heart failure assessed based on plasma B-type natriuretic peptide (BNP) levels negatively impacts activity of daily living in patients with hip fracture. PLoS ONE 15:e0237387. https://doi.org/10.1371/journ al.pone. 0237387

17. Xie J, Qiu L, Tao Y et al (2019) Perioperative myocardial injury after noncardiac surgery: an easily ignored and challenging problem. Int J Cardiol 292:42. https://doi.org/10.1016/j.ijcard. 2019.04.059

18. Ponikowski P, Voors AA, Anker SD et al (2016) 2016 ESC Guidelines for the diagnosis and treatment of acute and chronic heart failure: the Task Force for the diagnosis and treatment of acute and chronic heart failure of the European Society of Cardiology (ESC). Developed with the special contribution of the Heart Failure Association (HFA) of the ESC. Eur J Heart Fail 18:891-975. https://doi.org/10.1002/ejhf.592

19. Patel JN, Klein DS, Sreekumar S et al (2020) Outcomes in multidisciplinary team-based approach in geriatric hip fracture care: a systematic review. J Am Acad Orthop Surg 28:128-133. https://doi.org/10.5435/JAAOS-D-18-00425

20. Greenstein AS, Gorczyca JT (2019) Orthopedic surgery and the geriatric patient. Clin Geriatr Med 35:65-92. https://doi.org/10. 1016/j.cger.2018.08.007

21. Erickson KLB (2020) Innovations in care of the elderly hip fracture patient; a nightmare no more. Nurs Clin North Am 55:149-161. https://doi.org/10.1016/j.cnur.2020.02.010

22. Zanker J, Duque G (2017) Rapid geriatric assessment of hip fracture. Clin Geriatr Med 33:369-382. https://doi.org/10. 1016/j.cger.2017.03.003

23. Zhou K, Lai Y, Wang Y et al (2020) Comprehensive pulmonary rehabilitation is an effective way for better postoperative outcomes in surgical lung cancer patients with risk factors: a propensity score-matched retrospective cohort study. Cancer Manag Res 12:8903-8912. https://doi.org/10.2147/CMAR. S267322

24. Vincent JL (2019) Fluid management in the critically ill. Kidney Int 96:52-57. https://doi.org/10.1016/j.kint.2018.11.047

25. Liu VX, Rosas E, Hwang J et al (2017) Enhanced recovery after surgery program implementation in 2 surgical populations in an integrated health care delivery system. JAMA Surg 152:e171032. https://doi.org/10.1001/jamasurg.2017.1032

26. Zhou $\mathrm{H}, \mathrm{Hu} \mathrm{Y}, \mathrm{Li} \mathrm{X}$ et al (2018) Assessment of the risk of venous thromboembolism in medical inpatients using the Padua prediction score and caprini risk assessment model. J Atheroscler Thromb 25:1091-1104. https://doi.org/10.5551/jat.43653

27. De Vincentis A, Behr AU, Bellelli G et al (2021) Orthogeriatric co-management for the care of older subjects with hip fracture: recommendations from an Italian intersociety consensus. Aging Clin Exp Res 33:2405-2443. https://doi.org/10.1007/ s40520-021-01898-9 
28. Salazar J, Martinez MS, Chavez M et al (2014) C-reactive protein: clinical and epidemiological perspectives. Cardiol Res Pract 2014:605810. https://doi.org/10.1155/2014/605810

29. Williams ES, Shah SJ, Ali S et al (2008) C-reactive protein, diastolic dysfunction, and risk of heart failure in patients with coronary disease: Heart and Soul Study. Eur J Heart Fail 10:63-69. https://doi.org/10.1016/j.ejheart.2007.11.003

30. Cauley JA, Cawthon PM, Peters KE et al (2016) Risk factors for hip fracture in older men: the osteoporotic fractures in men study (MrOS). J Bone Miner Res 31:1810-1819. https://doi.org/10. 1002/jbmr.2836

31. de Sire A, Invernizzi M, Baricich A et al (2021) Optimization of transdisciplinary management of elderly with femur proximal extremity fracture: a patient-tailored plan from orthopaedics to rehabilitation. World J Orthop 12:456-466. https://doi.org/10. 5312/wjo.v12.i7.456

32. National Clinical Guideline C (2011) National institute for health and clinical excellence: guidance. In: The management of hip fracture in adults, vol London: Royal College of Physicians (UK)

33. Mak J, Wong E, Cameron I et al (2011) Australian and New Zealand society for geriatric medicine. Position statement-orthogeriatric care. Austral J Ageing 30:162-169. https://doi.org/10.1111/j. 1741-6612.2011.00557.x

34. Roche JJ, Wenn RT, Sahota O et al (2005) Effect of comorbidities and postoperative complications on mortality after hip fracture in elderly people: prospective observational cohort study. BMJ 331:1374. https://doi.org/10.1136/bmj.38643.663843.55

35. Ponikowski P, Voors AA, Anker SD et al (2016) 2016 ESC Guidelines for the diagnosis and treatment of acute and chronic heart failure: the Task Force for the diagnosis and treatment of acute and chronic heart failure of the European Society of Cardiology (ESC)Developed with the special contribution of the Heart Failure Association (HFA) of the ESC. Eur Heart J 37:2129-2200. https:// doi.org/10.1093/eurheartj/ehw128

36. Clerico A, Emdin M (2004) Diagnostic accuracy and prognostic relevance of the measurement of cardiac natriuretic peptides: a review. Clin Chem 50:33-50. https://doi.org/10.1373/clinchem. 2003.024760

37. Velagaleti RS, Gona P, Larson MG et al (2010) Multimarker approach for the prediction of heart failure incidence in the community. Circulation 122:1700-1706. https://doi.org/10.1161/ CIRCULATIONAHA.109.929661

38. Nishikimi T, Kuwahara K, Nakao K (2011) Current biochemistry, molecular biology, and clinical relevance of natriuretic peptides. J Cardiol 57:131-140. https://doi.org/10.1016/j.jjcc.2011.01.002
39. Zhang Y, Luo Y, Nijiatijiang G et al (2019) Correlations of changes in brain natriuretic peptide (BNP) and cardiac troponin I (cTnI) with levels of C-reactive protein (CRP) and TNF- $\alpha$ in pediatric patients with sepsis. Med Sci Monit 25:2561-2566. https:// doi.org/10.12659/MSM.912318

40. Chan DS, Bandera EV, Greenwood DC et al (2015) Circulating C-reactive protein and breast cancer risk-systematic literature review and meta-analysis of prospective cohort studies. Cancer Epidemiol Biomark Prev 24:1439-1449. https://doi.org/10.1158/ 1055-9965.EPI-15-0324

41. Markozannes G, Koutsioumpa C, Cividini S et al (2021) Global assessment of C-reactive protein and health-related outcomes: an umbrella review of evidence from observational studies and Mendelian randomization studies. Eur J Epidemiol 36:11-36. https:// doi.org/10.1007/s10654-020-00681-w

42. Fakler JK, Grafe A, Dinger J et al (2016) Perioperative risk factors in patients with a femoral neck fracture - influence of 25-hydroxyvitamin D and C-reactive protein on postoperative medical complications and 1-year mortality. BMC Musculoskelet Disord 17:51. https://doi.org/10.1186/s12891-016-0906-1

43. Neuerburg C, Forch S, Gleich J et al (2019) Improved outcome in hip fracture patients in the aging population following co-managed care compared to conventional surgical treatment: a retrospective, dual-center cohort study. BMC Geriatr 19:330. https:// doi.org/10.1186/s12877-019-1289-6

44. Sabharwal S, Wilson H (2015) Orthogeriatrics in the management of frail older patients with a fragility fracture. Osteoporos Int 26:2387-2399. https://doi.org/10.1007/s00198-015-3166-2

45. Yin M, Yan Y, Fan Z et al (2020) The efficacy of enhanced recovery after surgery (ERAS) for elderly patients with intertrochanteric fractures who received surgery: study protocol for a randomized, blinded, controlled trial. J Orthop Surg Res 15:91. https:// doi.org/10.1186/s13018-020-01586-w

46. Tarazona-Santabalbina FJ, Ojeda-Thies C, Figueroa Rodriguez $\mathrm{J}$ et al (2021) Orthogeriatric management: improvements in outcomes during hospital admission due to hip fracture. Int J Environ Res Public Health. https://doi.org/10.3390/ijerph18063049

Publisher's Note Springer Nature remains neutral with regard to jurisdictional claims in published maps and institutional affiliations.

\section{Authors and Affiliations}

\section{Mingming $\mathrm{Fu}^{1}$ · Yaqian Zhang ${ }^{1}$ · Junfei Guo ${ }^{2}$. Yuqi Zhao ${ }^{1}$. Zhiyong Hou ${ }^{2,3} \cdot$ Zhiqian Wang $^{1} \cdot$ Yingze Zhang $^{2,3,4}$}

1 Department of Geriatric Orthopedics, The Third Hospital of Hebei Medical University, Shijiazhuang, Hebei 050051, People's Republic of China

2 Department of Orthopaedic Surgery, The Third Hospital of Hebei Medical University, Shijiazhuang, Hebei 050051, People's Republic of China
3 NHC Key Laboratory of Intelligent Orthopeadic Equipment (The Third Hospital of Hebei Medical University), Hebei, People's Republic of China

4 Chinese Academy of Engineering, Beijing 100088, People's Republic of China 